\title{
Conditional reconstruction: An alternative strategy in digital rock physics
}

\author{
Sadegh Karimpouli ${ }^{1}$ and Pejman Tahmasebi ${ }^{2}$
}

\begin{abstract}
Digital rock physics (DRP) is a newly developed method based on imaging and digitizing of 3D pore and mineral structure of actual rock and numerically computing rock physical properties, such as permeability, elastic moduli, and formation factor. Modern high-resolution microcomputed tomography scanners are used for imaging, but these devices are not widely available, and 3D imaging is also costly and it is a time-consuming procedure. However, recent improvements of 3D reconstruction algorithms such as crosscorrelation-based simulation and, on the other side, the concept of rock physical trends have provided some new avenues in DRP. We have developed a modified work flow using higher order statistical methods. First, a high-resolution 2D image is divided into smaller subimages. Then, different stochastic subsamples are generated based on the provided 2D subimages. Eventually, various rock physical parameters are calculated. Using
\end{abstract}

several subsamples allows extracting rock physical trends and better capturing the heterogeneity and variability. We implemented our work flow on two DRP benchmark data (Berea sandstone and Grosmont carbonate) and a thin-section image from the Grosmont carbonate formation. Results of realization models, pore network modeling, and autocorrelation functions for the real and reconstructed subsamples reveal the validity of the reconstructed models. Furthermore, the agreement between static and dynamic methods indicates that subsamples are representative volume elements. Average values of the subsamples' properties follow the reference trends of the rock sample. Permeability trends pass the actual results of the benchmark samples; however, elastic moduli trends find higher values. The latter can be due to image resolution and voxel size, which are generated by imaging tools and reconstruction algorithms. According to the obtained results, this strategy can be introduced as a valid and accurate method where an alternative method for standard DRP is needed.

\section{INTRODUCTION}

Rock physics studies are important for understanding and modeling the relationships between the rock samples and their physical properties. Conventional rock physics is based on theoretical and/or empirical relations under some assumptions and laboratory measurements. Modern microcomputed tomography $(\mu \mathrm{CT})$ scanners produce 3D high-resolution images of micropore structures of rock samples, which can be used in digital rock physics (DRP). Usually, a standard DRP is based on three steps (Dvorkin et al., 2011; Andrä et al., 2013a, 2013b; Blunt et al., 2013): (1) high-resolution 3D imaging, (2) image segmentation using image processing algorithms to delineate and separate pore space and mineral phases, and (3) simulation of physical properties, such as fluid flow, electrical current flow, and elastic deformation to quantify permeability, resistivity, and elastic-moduli/elastic-wave, respectively. Based on the reported comparisons between the rock properties produced by DRP methods and laboratory measurements, they indicate that DRP results are valid enough to be used in the petroleum industry (Dvorkin and Derzhi, 2012; Lopez et al., 2012; Madonna et al., 2012; Ceron et al., 2013; Ringstad et al., 2013; Jouini et al., 2014; Liubis and Harith, 2014).

However, an outstanding challenge in the DRP method is that $\mu \mathrm{CT}$ scanners are not available extensively. Moreover, producing a $3 \mathrm{D}$ image of a sample in the millimeter scale is very time-demanding and costly. These issues motivated us to develop alternative methods that can be used individually and/or along with standard DRP and laboratory analyses. One of these techniques is the stochastic-based $2 \mathrm{D}$ to $3 \mathrm{D}$ reconstruction method, which simulates 3D structure of pore space and mineral phases of rock sample using

\footnotetext{
Manuscript received by the Editor 4 May 2015; revised manuscript received 27 December 2015; published online 29 June 2016.

${ }^{1}$ University of Zanjan, Mining Engineering Group, Zanjan, Iran. E-mail: s.karimpouli@znu.ac.ir.

${ }^{2}$ University of Southern California, Mork Family Department of Chemical Engineering and Materials Science, Los Angeles, California, USA and California Institute of Technology, Division of Engineering and Applied Science, Mechanical and Civil Engineering, Pasadena, California, USA. E-mail: pejman@ caltech. edu.

(C) 2016 Society of Exploration Geophysicists. All rights reserved.
} 
a 2D image such as a thin section. Liang et al. (2000) use an algorithm based on morphological skeletonization, Talukdar and Torsaeter (2002) apply simulated annealing method, Keehm et al. (2003) use sequential indicator simulation to reconstruct 3D images, Okabe and Blunt $(2004,2005,2007)$ reconstruct a 3D pore space structure using multiple-point statistics and predicted permeability, and Latief et al. (2010) represent a model reconstruction based on crystallite properties and diagenetic parameters. In this study, however, we used a newly presented accurate reconstruction method introduced by Tahmasebi and Sahimi $(2012,2013)$ using crosscorrelationbased simulation (CCSIM). The main idea in the CCSIM is to use a crosscorrelation function along a 1D raster path and to combine it with efficient strategies to honor the continuity and pattern reproducibility to generate realizations of the porous medium that match the image (Tahmasebi et al., 2012, 2014).

Unlike Keehm et al. (2003) and Okabe and Blunt (2004, 2005, 2007), reconstruction studies due to high heterogeneity and variability within the extracted samples should not be restricted to one single sample. Besides, using very large 3D images of different samples can increase the computational time dramatically. For this reason, Dvorkin et al. (2011) and Dvorkin and Derzhi (2012) show that different small proportions of a large sample can represent a trend that is valid over a range of scales. In the laboratory, these trends are produced by measuring a significant number of samples. In standard DRP, these trends can be driven by subsampling of a large digital sample. This idea is implemented in our proposed reconstruction method in three ways: (1) 2D imaging from several thin sections, (2) simulating several 2D image from one single $2 \mathrm{D}$ thin-section image, and (3) dividing a large 2D image to some subimages and, then, reconstructing of the image or subimages to generate subsamples. We selected the latter approach due to some computational limitations, and generated several subsamples using the CCSIM reconstruction method. Then, the subsequent steps remain as standard DRP, including segmentation and computation of rock physical properties of subsamples and finding corresponding rock physical trends.

Following this section, we describe our proposed method in detail, and then we implement it on two DRP benchmark data represented by Andrä et al. (2013a) to verify our obtained results by this method with standard DRP and laboratory measurements.

\section{METHODOLOGY}

Standard DRP steps are represented in detail by Andrä et al. (2013a, 2013b). They include (1) 3D imaging using high-resolution $\mu \mathrm{CT}$ scanner, (2) segmentation of image, and (3) rock parameter computation. In this paper, the aim is to present an efficient alternative method to overcome difficulties of producing a 3D image of a rock sample. In fact, different $3 \mathrm{D}$ stochastic models based on one or a few $2 \mathrm{D}$ images are used instead of $3 \mathrm{D}$ real images that, in essence, are very time-demanding and costly.

As mentioned, different subsamples for capturing the heterogeneity and variability are used in this study. This allows us to find the existence trends properly. Figure 1 shows a graphical flowchart of the proposed method. According to this figure, the first step of standard DRP (Dvorkin et al., 2011; Andrä et al., 2013a, 2013b) is replaced by three phases (Figure 1a.I-a.III), but the second (Figure 1b) and third (Figure 1c) steps are as standard DRP.

\section{D imaging}

The first step in DRP is imaging. However, in this method, instead of using high-resolution $3 \mathrm{D}$ images, we rely on a single $2 \mathrm{D}$ image. A simple thin section, for example, can be used to obtain a 2D image (Figure 1a.I). This image can be captured easily by a conventional laboratory microscope and a high-resolution camera,

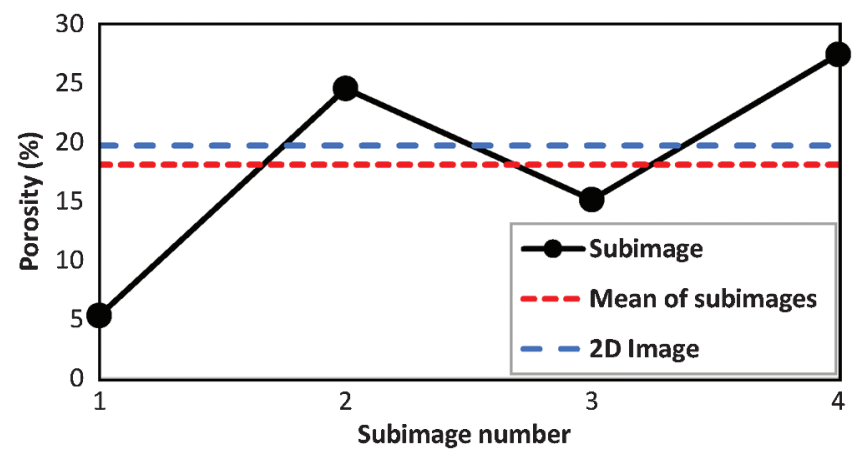

Figure 2. Porosity values of image, subimages, and mean value of subimages of Figure 1a.I and 1a.II.
a.I)

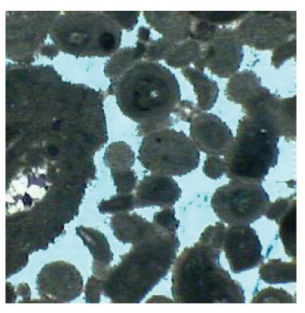

a.II)
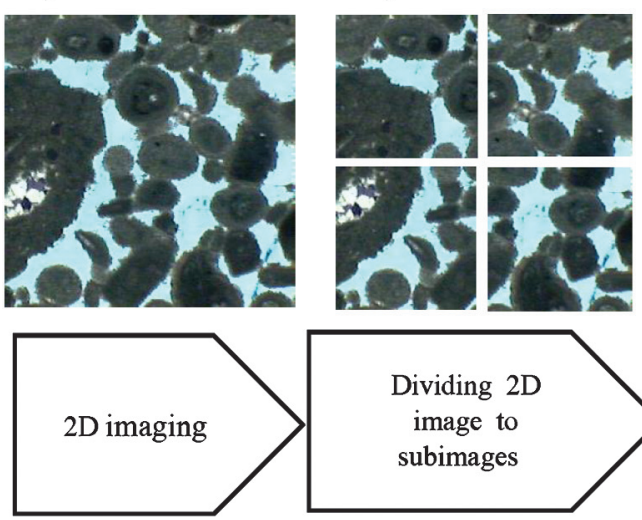

a.III)

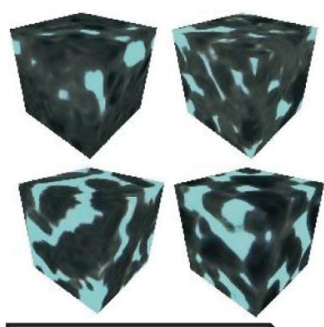

$2 \mathrm{D}$ to $3 \mathrm{D}$

reconstruction of subimages b)

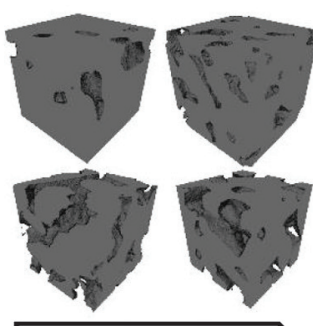

Segmentation of subsamples c)

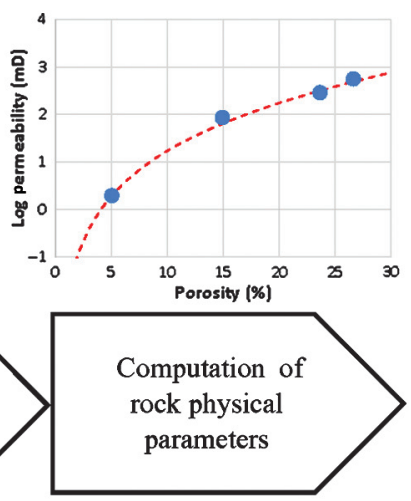

Figure 1. (a.I) 2D imaging (from, for example, a thin section), (a.II) dividing 2D image to subimages, (a.III) 2D to 3D reconstruction of subimages, (b) segmentation, and (c) computation of rock parameters from subsamples and, then, finding rock physical trends. 
which are widely available. Other sources of high-resolution 2D image such as scanning electron microscopy can be used as well.

\section{Dividing a 2D image to subimages}

To find a trend of rock physical parameters, several samples or subsamples are needed. However, because the aim of this paper is using a 2D image, different approaches can be used potentially to

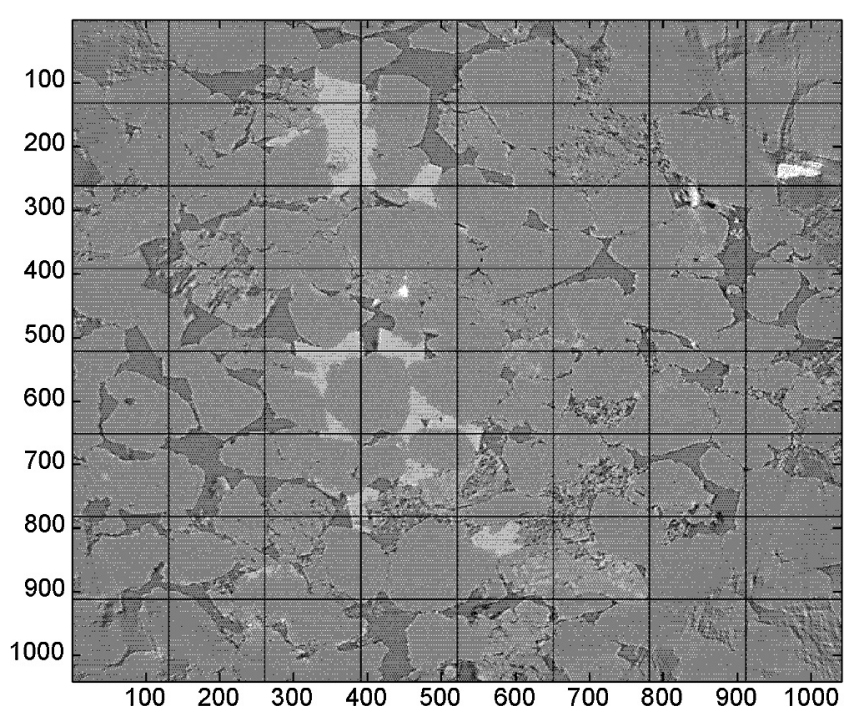

Figure 3. Divided 2D image number 150 selected from 3D $\mu \mathrm{CT}$-scan image of Berea sandstone. reconstruct 3D subsamples from $2 \mathrm{D}$ image(s). One way is to prepare several 2D thin sections. In practice, this is an elegant idea because more scenarios of real rock samples are captured. However, providing different thin sections can be very time-demanding, whereas the aim of this paper is presenting an equivalent method that can keep us away from this step. Another approach is to use a single 2D image, implement the CCSIM algorithm and produce several 2D images stochastically, and then reconstruct 3D subsamples. However, using this method does not to let some external patterns to appear in the simulation. An optimal solution, which we used in this study, is to use a large 2D image and divide it to subimages with a size of $128 \times 128$ pixels (the same as producing subsamples in Dvorkin et al., 2011). This approach has the advantage of using several direct 2D images with the real rock structures that can exhibit the heterogeneity and complexity in the sample more realistically. To this end, the initial large $2 \mathrm{D}$ image can be divided regularly to subimages and/or one can select some of subsets randomly. Although it is expected to get similar results using each subimage, in this study, we produced subimages by regularly dividing the $2 \mathrm{D}$ image.

It should be noted that dividing the input $2 \mathrm{D}$ image may produce some subimages that are not representative of the real sample even though they are extracted from a real image of the rock. This issue in an image with low entropy is remarkable. Therefore, it is recommended to choose those subimages that are more similar to the real rock structure. As such, we expect rock physical properties to be changed around an average value close to the real value (Dvorkin et al., 2011). For example, as it is illustrated in Figure 2, the porosity of the thin-section image in Figure 1a.I is $19.7 \%$, whereas the porosity of subimages (Figure 1a.II) varies from $5.3 \%$ to $27.4 \%$ with a a)

Real subsample

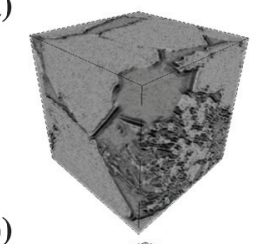

b)

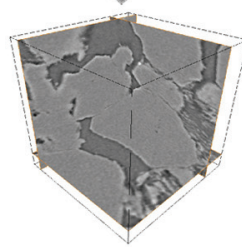

c)

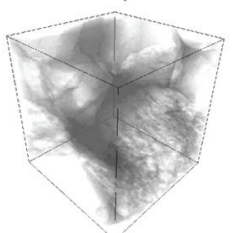

d)

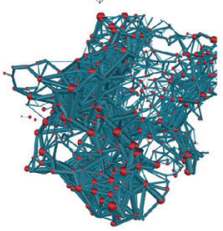

Reconstructed subsample
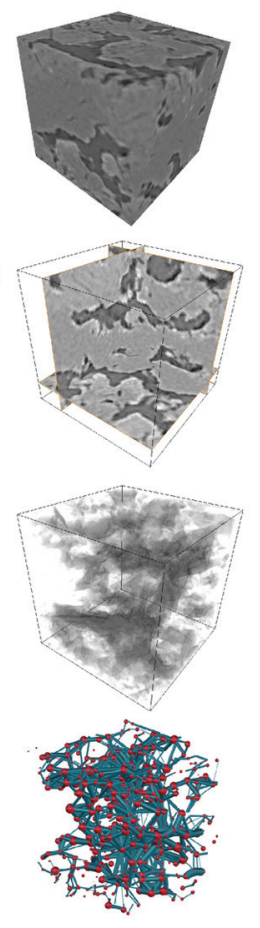

More realizations of subsample 3D cube
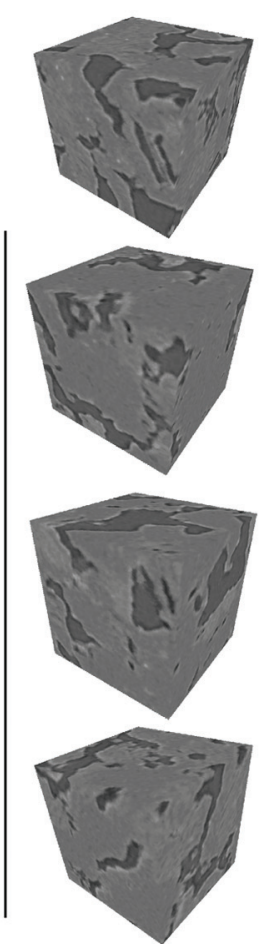

Orthogonal slices
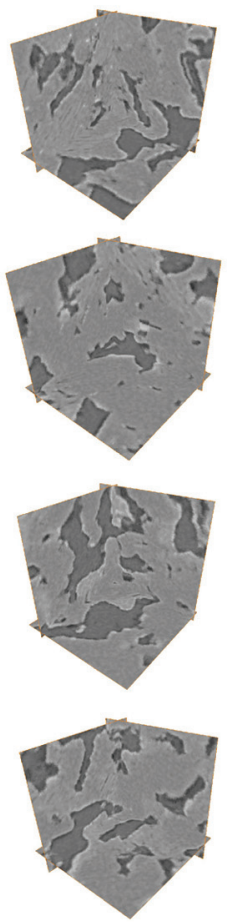

Figure 4. Real and realizations of reconstructed model of Berea sandstone subimage number 18: (a) 3D view, (b) orthogonal slices in $x-, y$-, and $z$-directions, (c) segmented pore structure, and (d) pore network modeling. 
mean of $18.1 \%$, which is very close to the porosity of the input image $(19.7 \%)$.

\section{D to 3D reconstruction of subimages}

The CCSIM algorithm (Tahmasebi et al. 2012, 2014) is used as the groundwork of reconstructing of a 3D model from a 2D image. In its very basic form, the CCSIM starts from a corner in the simulation grid and randomly selects the first pattern from the provided input image. A small band of overlap is considered for preserving the continuity of the current patterns and the next segment that is going to be inserted into the simulation grid. Thus, the next pattern is identified using the selected overlap. It should be noted that we are not interested in identifying the best matching pattern; rather, an ensemble of candidate patterns are selected, and one of them is randomly chosen as the final pattern. The algorithm proceeds until the simulation grid is filled. Clearly, one of the main factors in the CCSIM that controls the quality of the generated realizations is the number of candidate patterns. One can use a high number of candidates, which leads to increasing the variability and also decreasing the quality of the realization. Therefore, there is always a trade-off between the number of candidate patterns and the realization's quality/variability. Due to the high complexity of the examples in this paper, we used five candidates.

However, the basic algorithm is used in a different way. First, the original image is set as the first layer in the most bottom part of the 3D model. Then, the other four frames (i.e., front, left, back, and right) are generated using the conditional CCSIM algorithm. These frames keep the exterior continuity of the 3D model. Next, the optimal locations of conditional (i.e., hard) data for the second layer are determined according to Shannon entropy. Using this idea, a satisfactory vertical continuity and pattern reproduction can be achieved. It should be noted that each new layer is conditioned to the edges to preserve the continuity along the external farms. Then, the next layer can be generated using the extracted hard data. This procedure continues until all layers are reconstructed. Eventually, the $3 \mathrm{D}$ reconstructed model is formed by stacking the entire sequentially generated layers. Besides, to induce more variability and heterogeneity to the final 3D reconstructed model, one also can use various 2D images along each axis (Tahmasebi et al. 2015a, 2015b). However, in this study, one image is used, and an isotropic assumption is made in the $3 \mathrm{D}$ model. More technical information regarding this algorithm is provided in Tahmasebi and Sahimi (2012, 2013, 2015).

Table 1. Important parameters of real and reconstructed Berea subsample number 18.

\begin{tabular}{lcc}
\hline & $\begin{array}{c}\text { Real } \\
\text { subsample }\end{array}$ & $\begin{array}{c}\text { Reconstructed } \\
\text { subsample }\end{array}$ \\
\hline Porosity (\%) & 25.8 & 24.2 \\
Average pore radius (mm) & $0.12 \times 10^{-1}$ & $0.16 \times 10^{-1}$ \\
Average throat radius (mm) & $0.68 \times 10^{-2}$ & $0.99 \times 10^{-2}$ \\
Average pore volume $\left(\mathrm{mm}^{3}\right)$ & $0.12 \times 10^{-1}$ & $0.15 \times 10^{-1}$ \\
Average throat volume $\left(\mathrm{mm}^{3}\right)$ & $0.71 \times 10^{-1}$ & $0.52 \times 10^{-1}$ \\
Average coordination number & 7.9 & 4.45 \\
\hline
\end{tabular}

\section{Segmentation}

Segmentation refers to delineation and separation of pore space and mineral phases (Figure 1b). Each voxel of subsamples is labeled according to its corresponding phase, which is essential for numerically computation of physical properties of subsamples. Segmentation is carried out either by manually imaging histogram thresholding (Madonna et al., 2012) or by some image processing algorithms, such as reference segmentation (visual science group [VSG]), Stanford segmentation, and Kongju segmentation (Andrä et al., 2013a). These algorithms usually contain advanced image
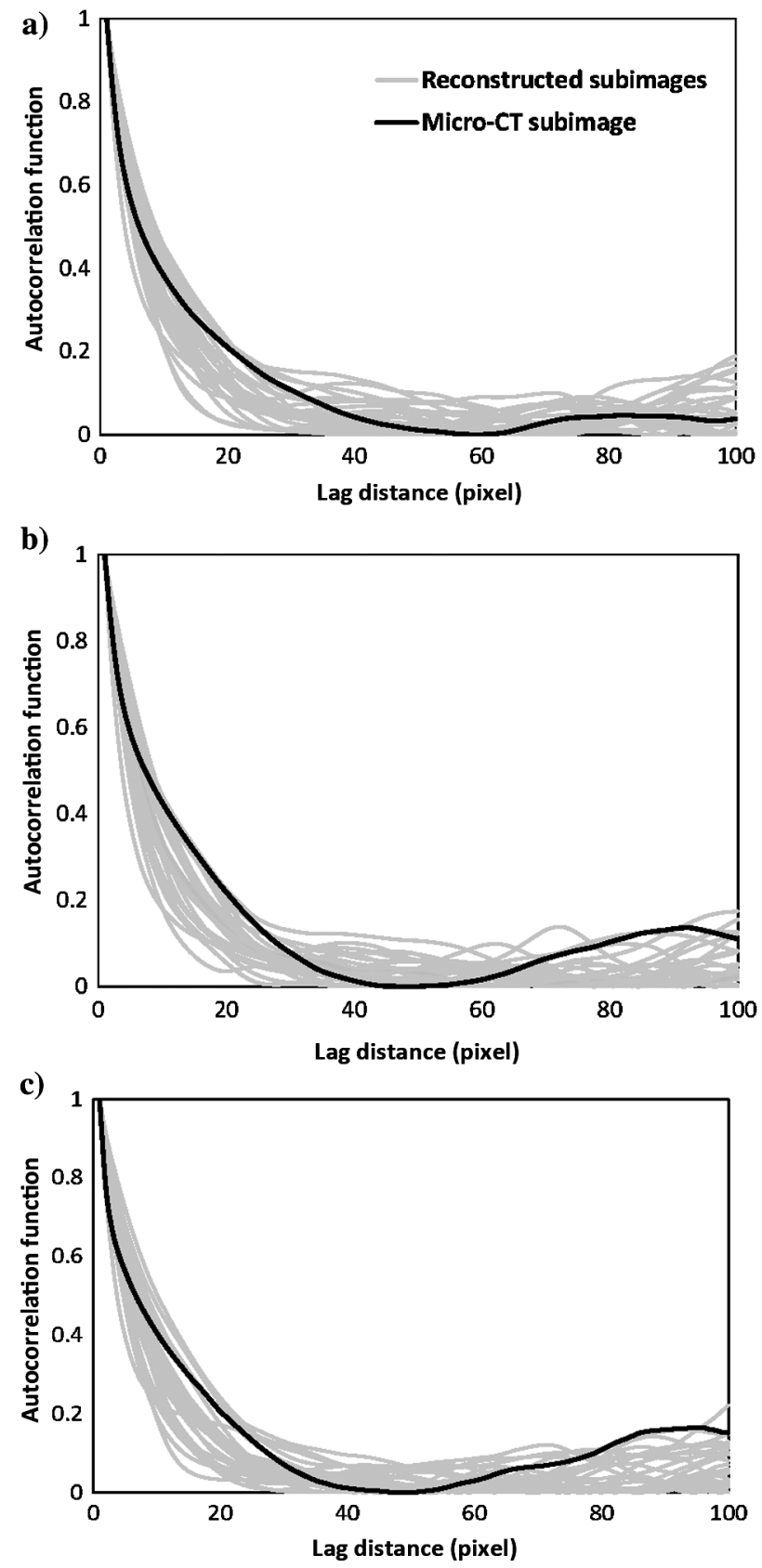

Figure 5. Autocorrelation functions computed for subimage number 18 and 30 realizations of the reconstructed model in (a) $x$-, (b) $y$ , and (c) $z$-directions. 
processing, such as spatial filtering, noise reduction, artifact removal, multiband thresholding, morphological operations, and cluster analysis (Andrä et al., 2013a).

Andrä et al. (2013b) use the VSG for image segmentation, whereas in this study a simple manually thresholding method was used (see Figure 1b). Clustering-based thresholding methods are usually classified as one of the most efficient thresholding techniques (Sezgin and Sankur, 2004). In this method, after detecting various minerals and pore phases in the histogram of the input image, a Gaussian mixture model (GMM) (McLachlan and Peel, 2000) with $n$ components is fitted for clustering, where $n$ is the number of phases. Then, posterior probabilities of the components are used to select an approximation for the cutoff value that can be used to separate pore phase from the minerals phases. Generally, based on the available evidences in the mineral distribution, the final cutoff value is manually determined. In the next section, some of the differences between this method and other automated processes are further demonstrated. One can segment the initial 2D image before $3 \mathrm{D}$ reconstruction. In other words, after dividing the $2 \mathrm{D}$ image into subimages, each subimage is segmented, and then a binary image is reconstructed to generate a $3 \mathrm{D}$ segmented subsample.

\section{Rock parameters of subsamples}

The most important part of DRP work flow is computing and simulating the physical parameters of rock. In this study, three parameters, elastic-wave (P- and S-wave) velocities and permeability, are studied. There are various algorithms for this purpose, which were classified and introduced by Andrä et al. (2013b). However, different algorithms may produce different results if a sample is not a representative volume element (RVE) (Saenger et al., 2006). An RVE is defined as a size of sample when its response under uniform displacement (Dirichlet) boundary condition becomes the same as that under uniform stress (Neumann) boundary condition (Saenger et al., 2006; Mostaghimi et al., 2013). These boundary conditions produce the upper and lower band limits of elastic moduli, respectively. Therefore, to reach more reliable results, two different methods, namely, static and dynamic, are applied for the elastic-wave velocity computation. Any similarity between these two results can confirm the comprehensibility of the used RVE samples.

\section{Finite-element method for static elasticity}

To compute the static elastic moduli, the finite-element method (FEM) of Garboczi and Day (1995) is applied. This method solves the basic Hook's law equations of linear elasticity:

$$
\tau=\mathbf{C} \boldsymbol{c}
$$

$$
\boldsymbol{\varepsilon}=\left|\nabla \mathbf{u}+(\nabla \mathbf{u})^{t}\right|
$$

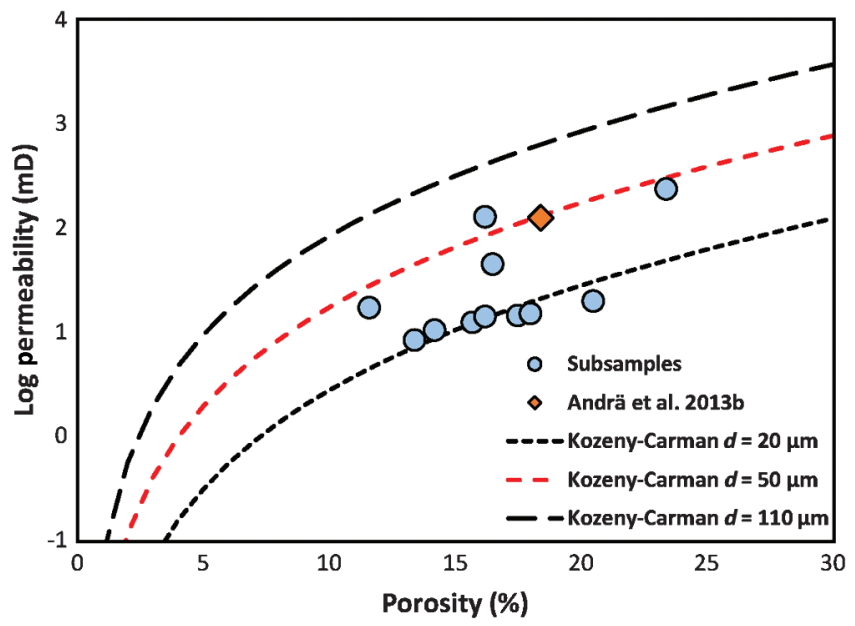

Figure 7. Computed permeability from subsamples with $128 \times$ $128 \times 128$ elements (circles) and the result computed by Andrä et al. (2013b) from the same sample with $1024 \times 1024 \times 1024$ elements (rectangle). Reference Kozeny-Carman trends are for grain size $110 \mu \mathrm{m}$ (long dashed line), $20 \mu \mathrm{m}$ (short dashed line), and $50 \mu \mathrm{m}$ (moderate dashed line).
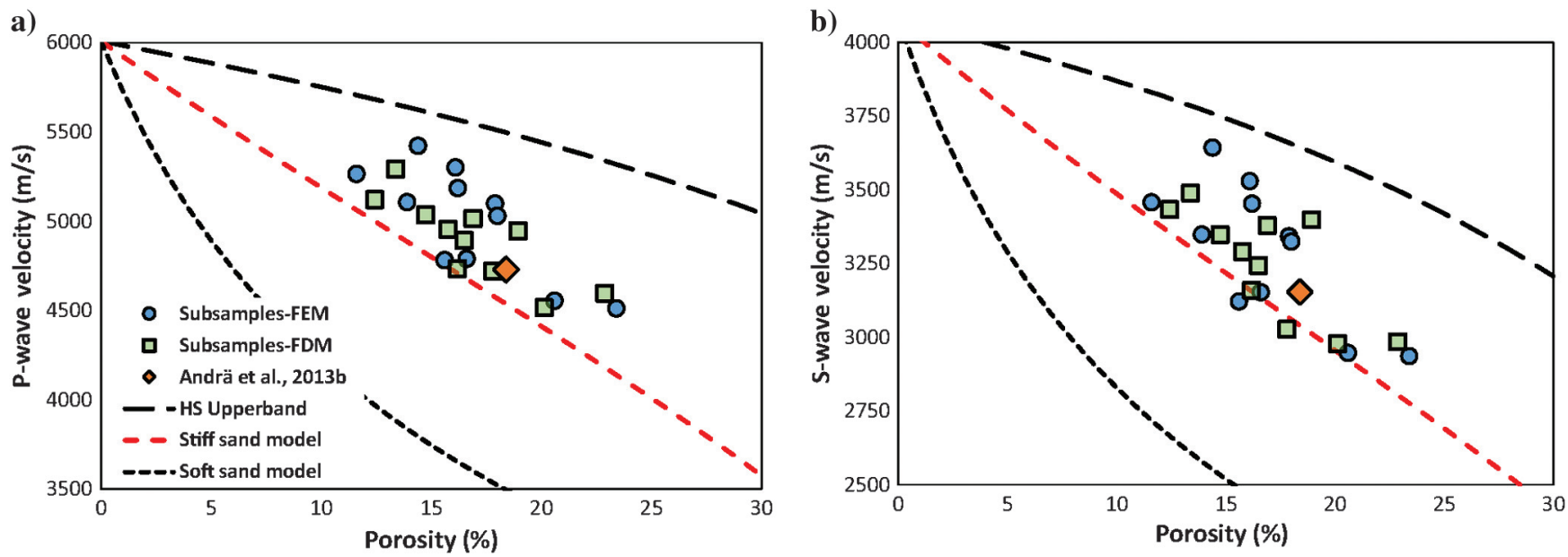

Figure 6. Computed (a) P- and (b) S-wave velocities from subsamples with $128 \times 128 \times 128$ elements using FEM (circles) and FDM (square) and the result computed by Andrä et al. (2013b) from the same sample with $1024 \times 1024 \times 1024$ elements (rectangle). Reference trends are the upper (long dashed line) and modified lower (short dashed line) HS bounds (soft sand model) and stiff sand model (moderate dashed line). 
where $\tau$ and $\varepsilon$ are the stress and strain tensor, respectively; $\mathbf{C}$ is the stiffness tensor; and $u$ is the displacement. In the FEM computation procedure, each voxel is considered as a trilinear element (FE mesh). Periodic boundary conditions are applied, and the elastic moduli of a material with different phases are calculated. In fact, phases are defined based on the difference in elastic moduli of each phase (void, fluid, and/or minerals). A uniform strain is used on a 3D sample, and therefore, local stresses are computed. The upper band limit of effective bulk $(K)$ and shear $(\mu)$ moduli is, finally, obtained by stress and strain components $\left(\tau_{i j}, \varepsilon_{i j}\right.$ where $i, j=$ $1,2,3$ ), using the following equations (Jouini et al., 2014):

$$
K=\left(\tau_{11}+\tau_{22}+\tau_{33}\right) /\left(3\left(\varepsilon_{11}+\varepsilon_{22}+\varepsilon_{33}\right)\right),
$$

$$
\mu=\left(\tau_{12} / \varepsilon_{12}+\tau_{13} / \varepsilon_{13}+\tau_{23} / \varepsilon_{23}\right) / 3 .
$$

Knowing the elastic moduli and density of the rock, one can easily compute the $\mathrm{P}$ - and $\mathrm{S}$-wave velocities using the following equations:

$$
\begin{gathered}
V_{\mathrm{P}}=\sqrt{\frac{K+\frac{4}{3} \mu}{\rho},} \\
V_{\mathrm{S}}=\sqrt{\frac{\mu}{\rho}} .
\end{gathered}
$$

\section{Finite-difference method for dynamic elasticity}

The finite-difference pulse propagation method (FDM) (Saenger et al., 2000; Saenger, 2008) is used for dynamic numerical simulation of elastic-wave propagation. In this approach, elastic-wave velocities are studied through heterogeneous materials in the longwavelength limit. The digitized rock sample is numerically embedded in a homogeneous elastic region with assigned elastic properties of the grain material. Periodic boundary conditions are

a)

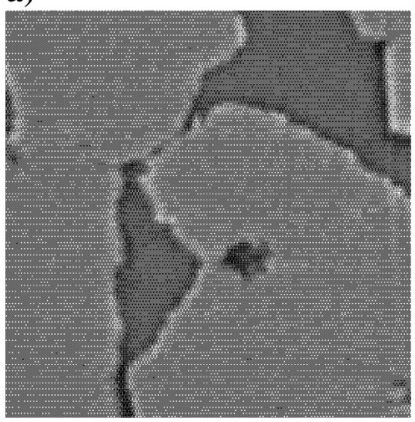

b)

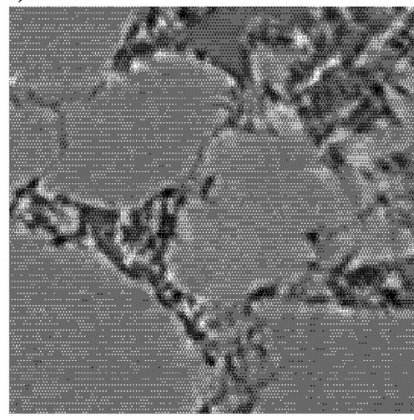

Figure 8. Subimage number (a) 33 with $d=50 \mu \mathrm{m}$ and (b) 40 with $d=20 \mu \mathrm{m}$. Different pore shape and pore throat size cause different permeability, and therefore, different trends. applied in the directions parallel to the wave propagation. A body-force plane source is used at the top of the model, in which the source signal is a broadband Gaussian pulse. The generated plane $\mathrm{P}$ - or S-waves can propagate through the model. With two planes of receivers at the top and bottom of the model, it is possible to measure the time delay of the peak amplitude of the mean plane wave caused by inhomogeneous structure of digitized rock sample. With the time delay (compared with a reference model), one can estimate the effective velocity of the P- and S-waves. These velocities are some values between the upper and lower band limits (Saenger et al., 2011, Andrä et al., 2013b).

\section{Lattice-Boltzmann method}

In this study, lattice-Boltzmann method (LBM) was used to compute absolute permeability (Ferreol and Rothman, 1995; Narváez

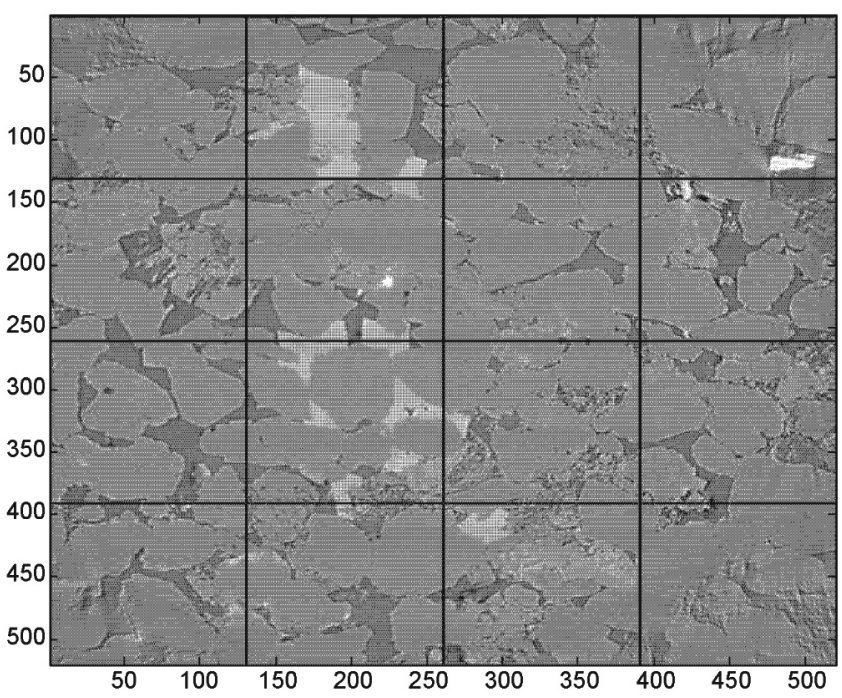

Figure 9. Resampling 2D image (Figure 3 ) by factor of 0.5 and dividing it to $16\left(=4^{2}\right)$ subimages with the size of $128 \times 128$ pixels.

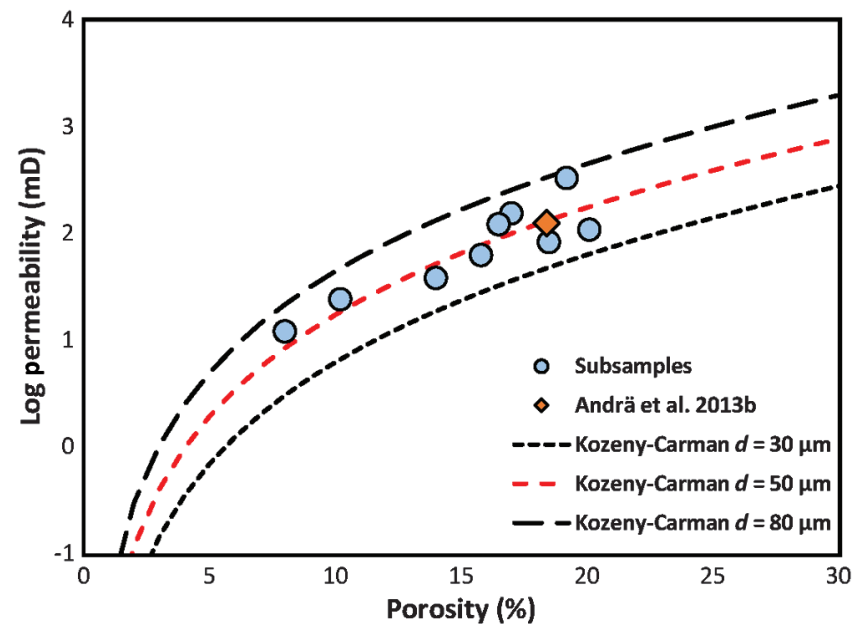

Figure 10. Same as Figure 7, but for resampled 2D image of Berea sandstone. Reference Kozeny-Carman trends are for grain size of $80 \mu \mathrm{m}$ (long dashed line), $30 \mu \mathrm{m}$ (short dashed line), and $50 \mu \mathrm{m}$ (moderate dashed line). 
et al. 2010). This method is accounted as an accurate technique to calculate fluid flow in rock pore structure.

The LBM simulates flow at the macroscopic scale according to local interactions among individual particles and recovering the Navier-Stokes equations. Boltzmann equation is solved at time $t$ and location of $r$ and, therefore, a volume averaged flux can be computed by the local flux. Then, the absolute permeability is calculated similar to a laboratory measurement considering a pressure head that is applied to a digital sample. After computing fluid flux,

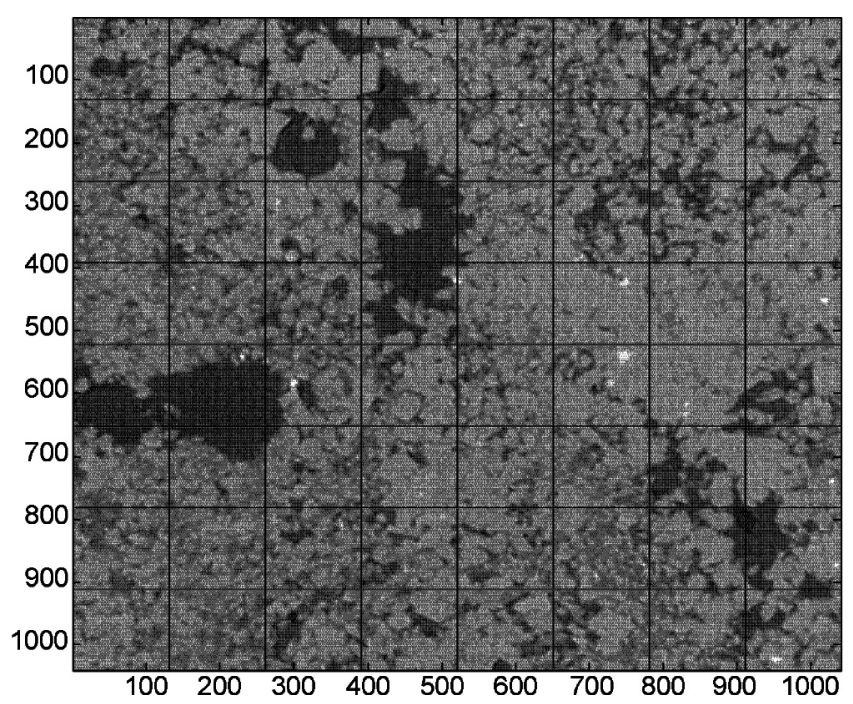

Figure 11. Divided 2D image number 900 of Grosmont carbonate, selected from 3D $\mu \mathrm{CT}$-scan data. permeability is calculated based on Darcy's law (Liubis and Harith 2014).

\section{DIGITAL ROCK PHYSICS BENCHMARKS}

Andrä et al. (2013a) introduce four benchmark data for DRP studies. To evaluate the proposed work flow, Berea sandstone and Grosmont carbonate are used in this study. In each case, we just used a 2D slice of 3D $\mu \mathrm{CT}$-scan image to predict rock physics parameters. To demonstrate the ability of this procedure as an alternative method in DRP, we also used a thin-section image of Grosmont carbonate as a $2 \mathrm{D}$ input image.

\section{Berea sandstone}

The Berea sandstone sample has $20 \%$ connected porosity with a permeability ranging from 200 to $500 \mathrm{mD}$. Petrographic micros-

Table 2. Important parameters of real and reconstructed Grosmont carbonate subsample number 64.

\begin{tabular}{lcc}
\hline & $\begin{array}{c}\text { Real } \\
\text { subsample }\end{array}$ & $\begin{array}{c}\text { Reconstructed } \\
\text { subsample }\end{array}$ \\
\hline Porosity (\%) & 22.7 & 21.7 \\
Average pore radius $(\mathrm{mm})$ & $0.45 \times 10^{-1}$ & $0.77 \times 10^{-1}$ \\
Average throat radius $(\mathrm{mm})$ & $0.25 \times 10^{-1}$ & $0.40 \times 10^{-1}$ \\
Average pore volume $\left(\mathrm{mm}^{3}\right)$ & 0.46 & 0.72 \\
Average throat volume $\left(\mathrm{mm}^{3}\right)$ & 1.01 & 1.61 \\
Average coordination number & 3.4 & 4.04 \\
\hline
\end{tabular}

a)

Real subsample
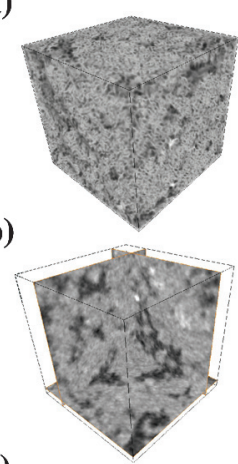

c)

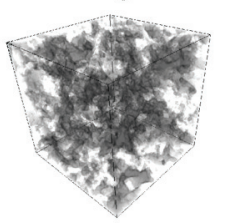

d)

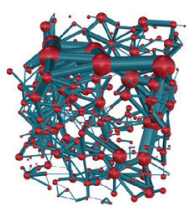

Reconstructed subsample
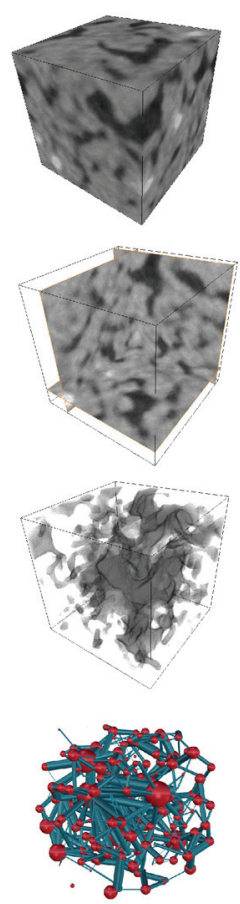

More realizations of subsample 3D cube
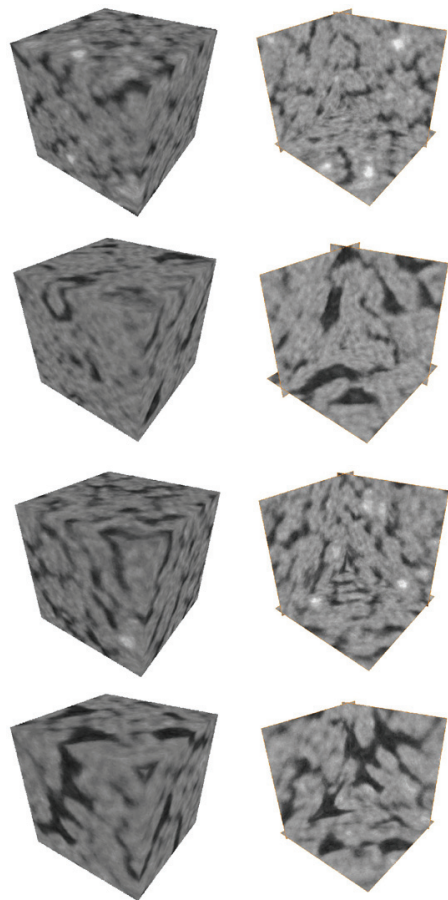

Figure 12. Real and realizations of reconstructed model of Grosmont carbonate subimage number 64: (a) 3D view, (b) orthogonal slices in $x$-, $y$-, and z-directions, (c) segmented pore structure, and (d) pore network modeling. 
copy and electron microprobe results show that the solid matrix material of this sample can be considered as an isotropic elastic solid. The Berea sandstone 3D $\mu$ CT-scan data consist of $10242 \mathrm{D}$ slices with $1024 \times 1024$ elements and a voxel size of $0.74 \mu \mathrm{m}$ (Madonna et al., 2012; Andrä et al., 2013a). One of the layers among all of 1024 images as a representative image of the Berea sandstone sample is selected. Then, based on the proposed method, the selected image is divided into $64\left(=8^{2}\right)$ subimages (Figure 3), which is considered as the input data later.

Figure 3 shows that all subimages are not obviously representative of whole rock sample. Most of them do not contain adequate patterns of pore structure such as those subimages in the corner and the center of the $2 \mathrm{D}$ image. Therefore, 11 representative subimages are selected. Then, each subimage is reconstructed, and 11 3D subsamples with $128 \times 128 \times 128$ voxels are produced. Next, these subsamples are segmented to separate mineral and pore phases. Finally, elastic-wave velocities and permeability are computed. A
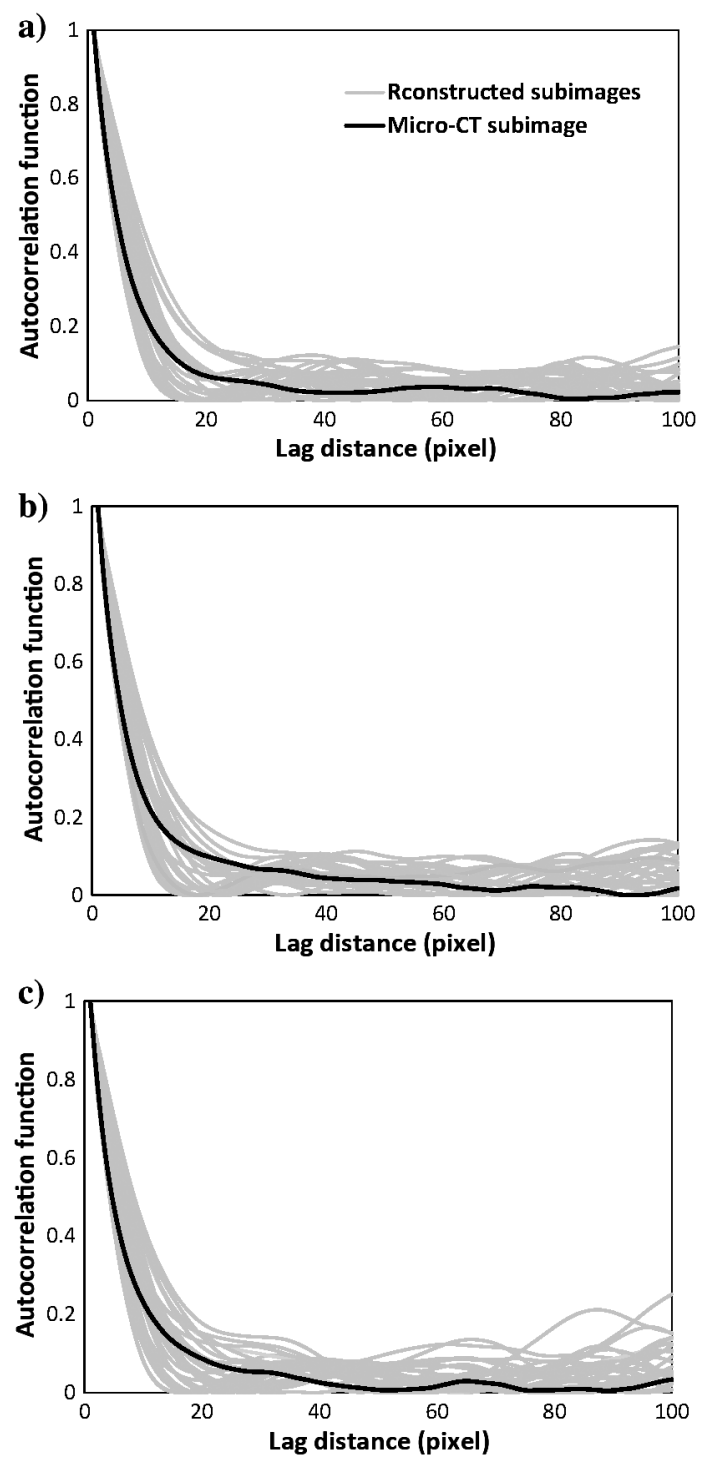

Figure 13. Autocorrelation functions calculated for 2D subimage and 30 realizations of $3 \mathrm{D}$ reconstructed subsample in (a) $x$-, (b) $y$-, and (c) $z$-directions. randomly selected subsample with its $3 \mathrm{D}$ reconstructed model for the sake of visual inspection is shown in Figure 4. The original sample and five different reconstructed models are shown in Figure 4. For having a closer look at the internal parts, one of the reconstructed models is taken and shown in $x$-, $y$-, and $z$-directions in Figure 4b. Segmentation and pore network modeling (Sochi and Blunt, 2008; Dong and Blunt, 2009) of the real and reconstructed subsamples can also depict the pore structure and porosity distribution, which are shown in Figure $4 \mathrm{c}$ and $4 \mathrm{~d}$, respectively. Table 1 numerically compares some important parameters of the real and reconstructed models of Figure 4 (Gao et al., 2015). The reconstructed image is a simulation of real sample; however, comparison between two sets of results confirms that they are in an acceptable range of variability. Autocorrelation functions (ACFs) (Box et al., 1994; Tahmasebi and Sahimi, 2012) are also computed for the real and reconstructed subimage in three directions ( $x, y$, and $z)$ to evaluate these models. To show the variability and validity of the subsamples, 30 realizations were produced, and then all directional ACFs were calculated. The results are shown in Figure 5 representing that the autocorrelation statistics is well-reproduced in the reconstructed model.

Using the proposed methods in section "Rock parameters of subsamples," P- and S-wave velocities are computed using the FEM and FDM methods, and the results are shown in Figure 6. To find the rock physical trends, these results are plotted versus porosity. Some other reference trends, such as the upper and modified lower Hashin-Shtrikman (HS) bounds (soft sand model) and stiff sand model (Dvorkin and Nur, 1996), are also illustrated for the sandstones. The similarity of results from the two different methods confirms that subsamples can be regarded as RVE. For the sake of comparison, computed parameters by Andrä et al. (2013b) for a same sample size of $1024 \times 1024 \times 1024$ elements are also plotted. As illustrated in Figure 4, subsample results change around a trend similar to reference trends, and they also pass close to the real result computed by Andrä et al. (2013b). These results confirm the validity of this method to generate a reliable and accurate 3D model based only on a single $2 \mathrm{D}$ image.

The permeability of the obtained $3 \mathrm{D}$ stochastic models is also computed, and the results are shown in Figure 7. These results are also plotted versus porosity to find the corresponding trend. In this case, reference trends are computed using the Kozeny-Carman relation for different grain sizes. Computed permeability for the same sample with $1024 \times 1024 \times 1024$ elements by Andrä et al. (2013b) is also plotted.

As illustrated in Figure 7, subsamples are showing two discernible trends: one with grain size of $20 \mu \mathrm{m}$ and the other one with grain size of $50 \mu \mathrm{m}$. The latter trend is the same as the actual result reported by Andrä et al. (2013b). To explore this circumstance, 2D subimages corresponding to each trend were further studied. The results show a bimodality of the pore shape and throat size for the existing subsamples. For instance, one subimage from each trend is displayed in Figure 8 . Although subimages share a similar porosity (16\%-18\%), they contain different pore shapes and throat sizes. Subimage number 33 (Figure 8a) contains vuggy-type porosity with a broader pore throat, whereas subimage number 40 (Figure 8 b) has so-called fracture patterns with a small pore throat. This causes subimage number 33 to have a higher permeability than subimage number 40 .

As shown in Figure 8a, the semifracture pore space has a negligible effect on permeability. Therefore, to obtain one single and 
valid permeability trend for the Berea sandstone, the 2D image is resampled by factor of 0.5 and divided into $16\left(=4^{2}\right)$ subimages with the size of $128 \times 128$ pixels (Figure 9). Thus, different subimages with larger voxel sizes $(2 \times 0.74=1.48 \mu \mathrm{m})$ and with more representative pores and mineral patterns are produced. In other words, permeability needs a larger subsample to be considered as the RVE (same as Mostagimi et al., 2013). Next, nine representative subimages are selected, and permeability is computed after reconstruction and segmentation, for each subsample. Figure 10 represents the results. It should be noted that all the subsamples fall along the Kozeny-Carman trend with grain size of $50 \mu \mathrm{m}$ passing from the same result presented by Andrä et al. (2013b).

It is worth mentioning that the purpose of the proposed stochastic method is not to reproduce an exact property for each subsample, but to generate a bunch of similar 3D models to cover the variability and uncertainty space (i.e., rock physical trends). The obtained results are in very good agreement with this objective.

\section{Grosmont carbonate}

The carbonate sample for this study is obtained from the Grosmont Formation in Alberta, Canada. This formation, with the age of upper Devonian, is a bitumen reservoir with four members: LG, UG1, UG2, and UG3 from the bottom. The sample selected from the UG2 member containing dolomite and karst breccia in composition. The lab-measured porosity is $21 \%$ porosity units, and permeability ranges from 150 to $470 \mathrm{mD}$. A $1024 \times 1024 \times 1024$ cube image was acquired using high-resolution X-ray computed tomography with a voxel edge length of $2.02 \mu \mathrm{m}$ (Andrä et al., 2013a). For this study, a 2D representative image with $1024 \times 1024$ elements is selected and divided it to $64\left(=8^{2}\right)$ subimages (see Figure 11).

To calculate rock physical parameters, 11 subimages are chosen as the representative subsamples. These subimages are first reconstructed and then segmented. Next, elastic-wave velocities and permeability are computed for each subsample. The 3D real subsample and five realizations of its reconstructed model are illustrated in Figure 12a. Three orthogonal slices in the $x$-, $y$-, and $z$-directions are also shown in Figure $12 \mathrm{~b}$ for one of realizations. Figure $12 \mathrm{c}$ represents the segmented pore space distribution through this subsample, and the pore network models (Sochi and Blunt, 2008; Dong and Blunt, 2009) are shown in Figure 12d. Upon visual inspection, the highly connected pore spaces are reproduced well. For a numerical comparison, different parameters of pore network for these models are computed and represented in Table 2 (Gao et al., 2015). The results of two sets are in an acceptable range of variability. Furthermore, the ACFs are calculated for the real sample and 30 realizations of the reconstructed subimage in the $x$-, $y$-, and $z$-directions. The results are presented in Figure 13. Autocorrelation results from 3D models are well-matched with actual data, which confirms the strength of the reconstruction work flow.

The results from $\mathrm{P}$ - and S-wave velocities calculation using the FEM and FDM are shown in Figure 14. For each parameter, the

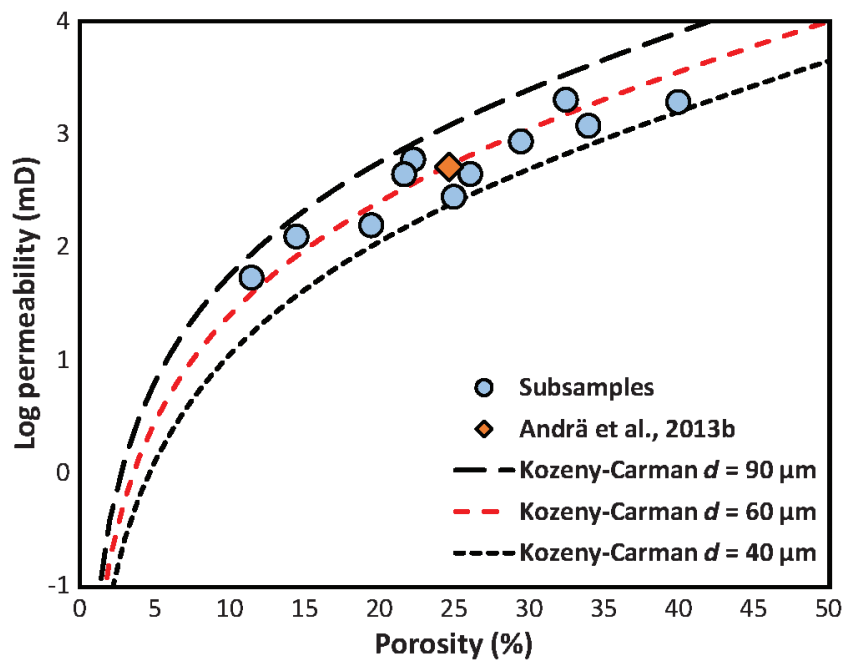

Figure 15. Same as Figure 7, but for a 2D image of Grosmont carbonate sample. Reference Kozeny-Carman trends are for grain size $90 \mu \mathrm{m}$ (long dashed line), $40 \mu \mathrm{m}$ (short dashed line), and $60 \mu \mathrm{m}$ (red dashed line).
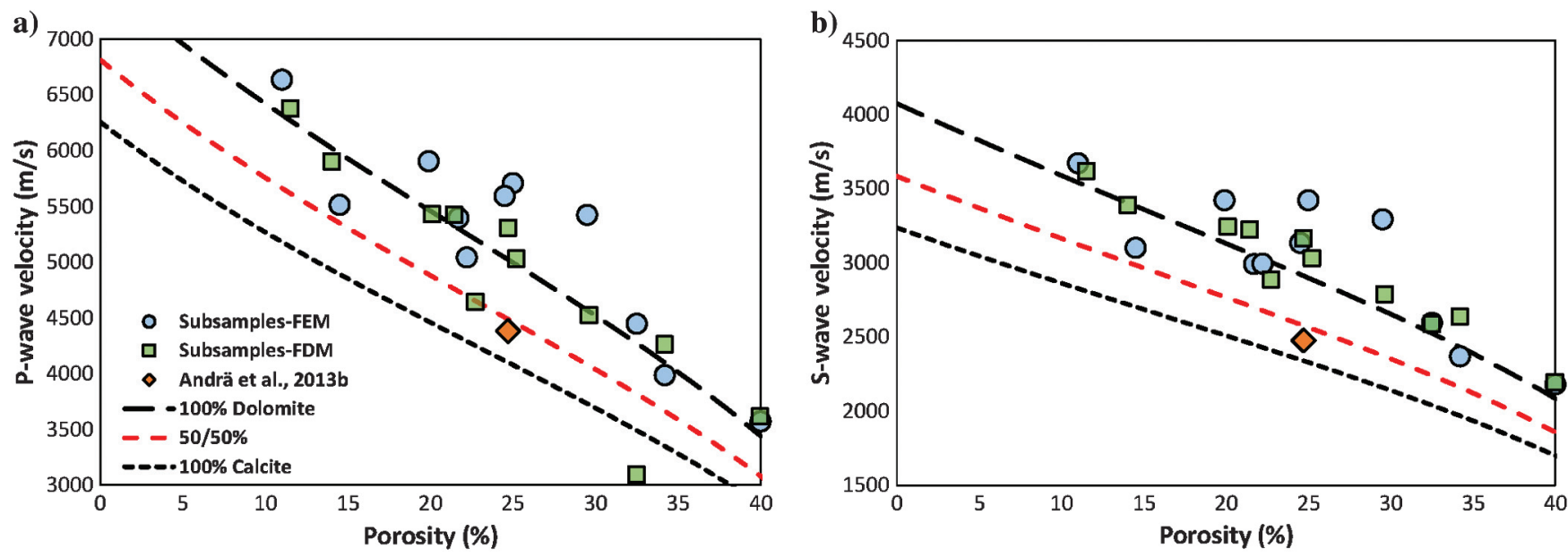

Figure 14. Computed (a) P- and (b) S-wave velocities from subsamples with $128 \times 128 \times 128$ elements using FEM (circles) and FDM (square) and the result computed by Andrä et al. (2013b) from the same sample with $1024 \times 1024 \times 1024$ elements (rectangle). Reference trends are stiff sand model for different mineralogical mixture: $100 \%$ calcite (long dashed line), $100 \%$ dolomite (short dashed line), and 50\%/50\% calcite and dolomite composition (red dashed line). 
reported results in Andrä et al. (2013b) are also plotted. The reference trend of the carbonate is modeled using the stiff sand model with a mineralogical mixture consisting of dolomite and calcite ( $100 \%$ calcite, $100 \%$ dolomite, and 50\%/50\% calcite and dolomite). For modeling, the carbonate rock critical porosity of 0.5 is assumed. As it is shown in Figure 14, all subsamples are located along trends similar to the reference trends. In addition, similar results from two numerical methods confirm that these subsamples are also RVE. In comparison with Berea sandstone, the carbonate RVE subsamples are larger than the sandstone RVE subsamples (same as Mostaghimi et al., 2013). Most of the generated subsample values are more than the reported values in Andrä et al. (2013b). This situation could be the result of using different resolutions. Indeed, elastic moduli are not only very sensitive to micro- and nanoporosities (Madonna et al., 2012; Andrä et al., 2013b; Saenger et al., 2014), but they also depend on porosity types in carbonate samples (Karimpouli et al., 2013). The bulk and shear moduli dramatically decrease with increasing fracture patterns that are usually below the resolution of the used image. Moreover, reconstructing the algorithm generates a 3D image with lower resolution than the main 2D image. Thus, some microstructures, especially microcracks and fracture-like pore spaces producing a stiffer sample, will be missed. This situation can be interpreted as a sample under a high pressure (Derzhi et al., 2011) that closes grain-to-grain contact and microcrack porosities, and therefore, makes a stiffer sample than the original rock.

Figure 15 shows the permeability results calculated for subsamples and the actual result (Andrä et al., 2013b). As is shown in this figure, the subsamples are well-distributed around the reference trend $(d=60 \mu \mathrm{m})$, which passes from the calculated permeability by Andrä et al., (2013b) for the same sample with $1024 \times 1024 \times$ 1024 elements.

\section{A thin section of Grosmont carbonate}

To show that the present method can be implemented by a highresolution $2 \mathrm{D}$ image, we found a $2 \mathrm{D}$ image from a thin section of the Grosmont Formation (Russel-Houston and Yuan, 2010;

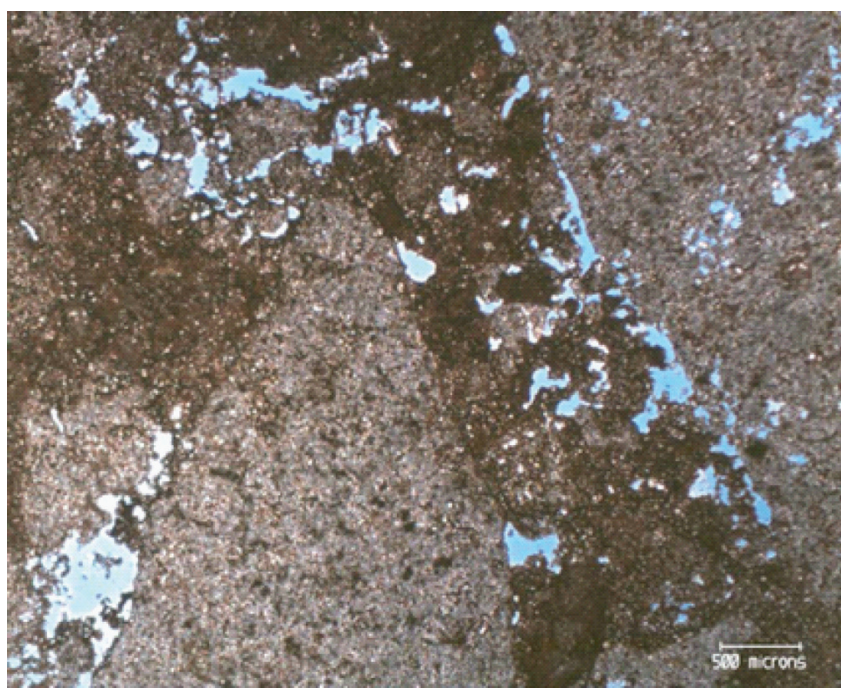

Figure 16. A 2D image from a thin section of Grosmont formation (from Russel-Houston and Yuan 2010).

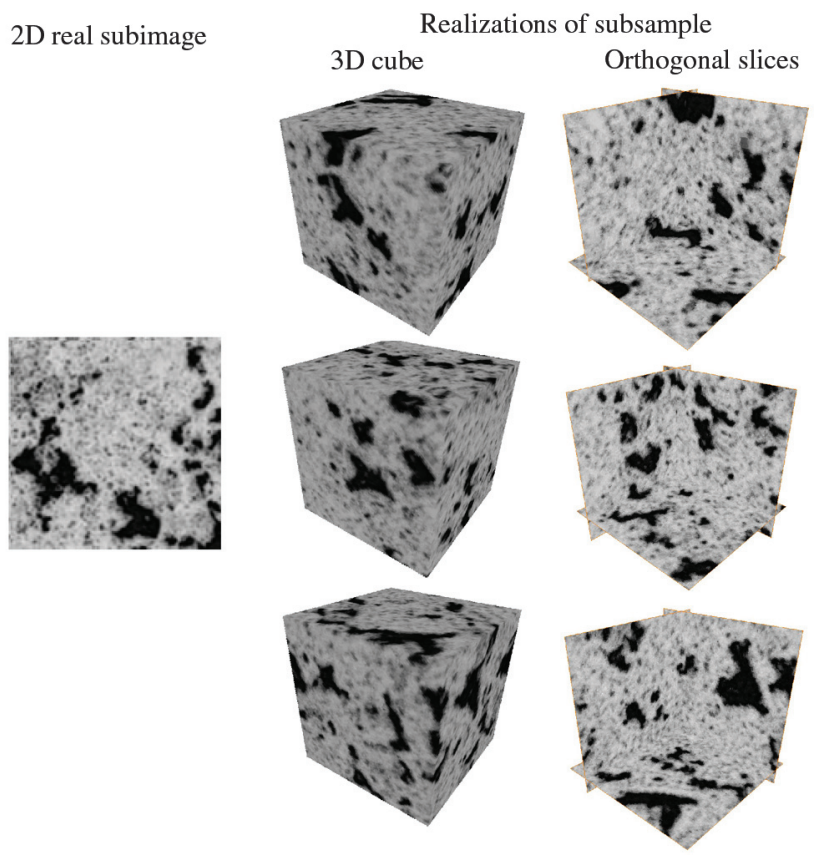

Figure 17. Real 2D subimage number 1 and three realizations of a reconstructed model of Grosmont carbonate.
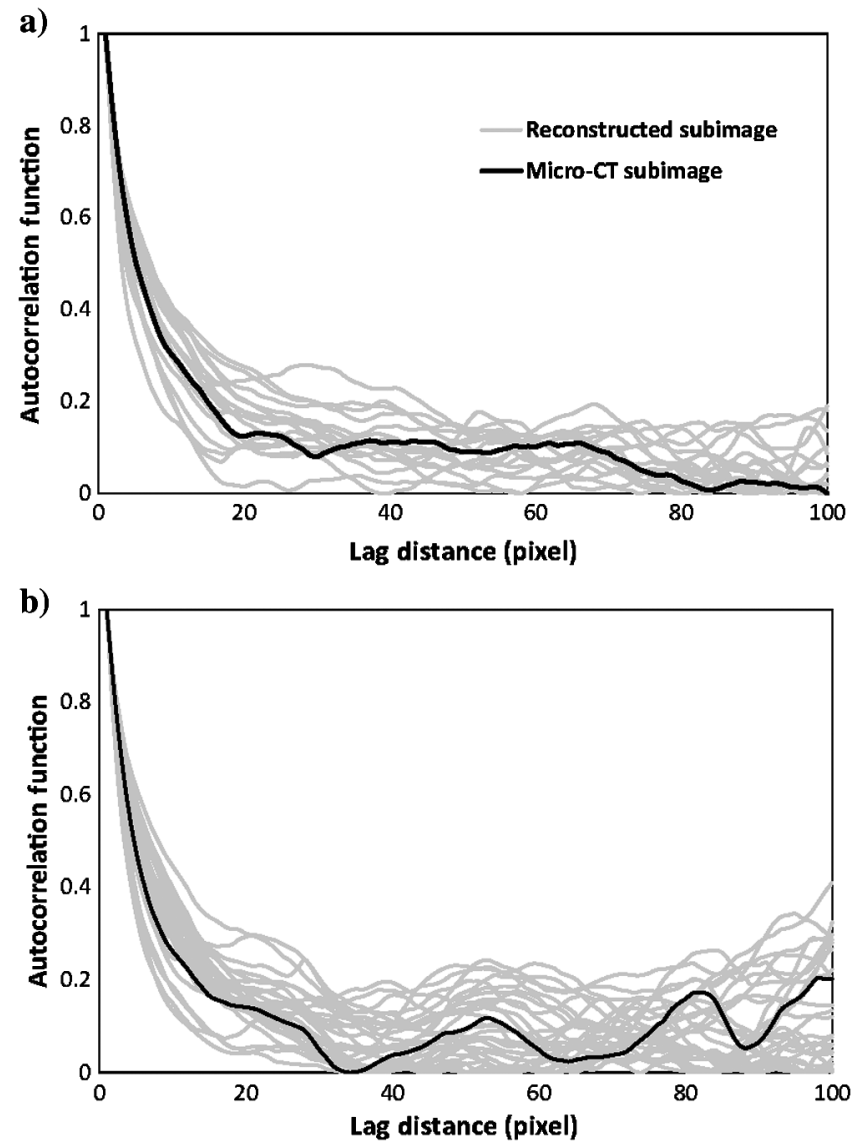

Figure 18. Autocorrelation functions calculated for a 2D subimage and 30 realizations of reconstructed subimages in (a) $x$ and (b) $y$. 
Figure 16). Based on the scale bar of the image, pixel size is $7.13 \mu \mathrm{m}$ which is large compares to the last samples. Images with a higher resolution can also be captured from thin sections, but because such a sample was not available for us, we had to go further with this $2 \mathrm{D}$ image. According to this method, the 2D image was divided, reconstructed, and segmented, and then the rock physical parameters were computed using simulated subsamples.

The input real 2D subimage and six 3D realizations are shown in Figure 17. Because the 3D real sample is not available, the results of the pore network computations are not presented for this example. However, the autocorrelation function is used for 30 realizations, and the real subimage and the results are presented in Figure 18. Once again, the results indicate that the reconstructed models are diverse enough, and they share a similar spatial correlation distribution with what exists in the 2D image. Thus, this makes the generated models to be well-applicable for the numerical simulation of rock physical parameters.

Figure 19 (same as Figure 14) shows the computed elastic-wave velocities of the Grosmont carbonate using the thin-section image. The resolution effect is also obvious, which caused a stiffer sample in both computational methods.

Permeability results, however, are more reliable (Figure 20) because they depend less on micropore structures, and their absence has an insignificant effect on permeability. To compare these results with the actual result (Andrä et al., 2013b), a benchmark image is resampled and a new image with adapted resolution to the thin section is generated. Then, the computed permeability on the adapted model is plotted for a more valid comparison, which is shown in Figure 20 . shows the posterior probabilities for three clusters: pore, mineral \#1 (quartz) and mineral \#2 (ankerite) in the Berea sandstone. According to these probabilities, one can manually find a proper cutoff value. Clearly, the calculated porosity by this method differs from other methods such as the VSG (Andrä et al., 2013b). Intuitively, this difference in porosity estimation has a direct effect on numeri-

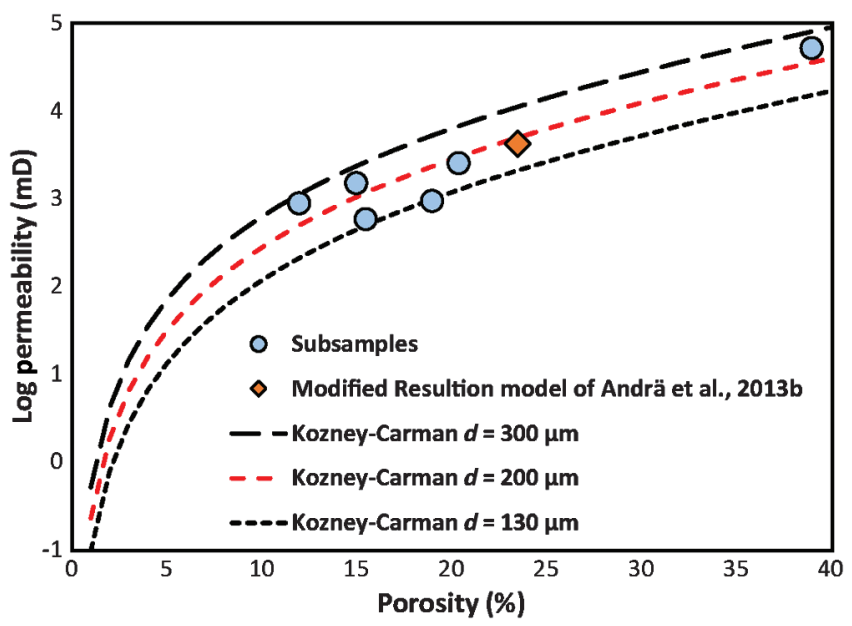

Figure 20. Computed permeability from subsamples with $128 \times 128 \times 128$ elements (circles) and from the same image with adapted resolution by Andrä et al. (2013b) (rectangle). Reference Kozeny-Carman trends are for grain size $300 \mu \mathrm{m}$ (long dashed line), $130 \mu \mathrm{m}$ (short dashed line), and $200 \mu \mathrm{m}$ (red dashed line).

\section{DISCUSSION}

In this study, two different methods of segmentation, namely automatic and manual clustering-based methods, were used. For performing the clustering-based method, after fitting an $n$ component GMM to a histogram of the image, posterior probabilities of the pore component were used to manually select a proper cutoff value for thresholding. For example, Figure 21
Table 3. Comparison of two image segmentation methods to estimate porosity and rock physical parameters.

\begin{tabular}{lcccc}
$\begin{array}{l}\text { Segmentation } \\
\text { method }\end{array}$ & $\begin{array}{c}\text { Porosity } \\
(\%)\end{array}$ & $\begin{array}{c}\text { Log-permeability } \\
(\mathrm{md})\end{array}$ & $\begin{array}{c}\text { P-wave velocity } \\
(\mathrm{m} / \mathrm{s})\end{array}$ & $\begin{array}{c}\text { S-wave velocity } \\
(\mathrm{m} / \mathrm{s})\end{array}$ \\
\hline $\begin{array}{l}\text { VSG (Andrä et al., } \\
\text { 2013b) }\end{array}$ & 21.95 & 156 & 4534 & 2951 \\
$\begin{array}{l}\text { Manual thresholding } \\
\text { (this study) }\end{array}$ & 23.12 & 241 & 4501 & 2932 \\
\hline
\end{tabular}

a)

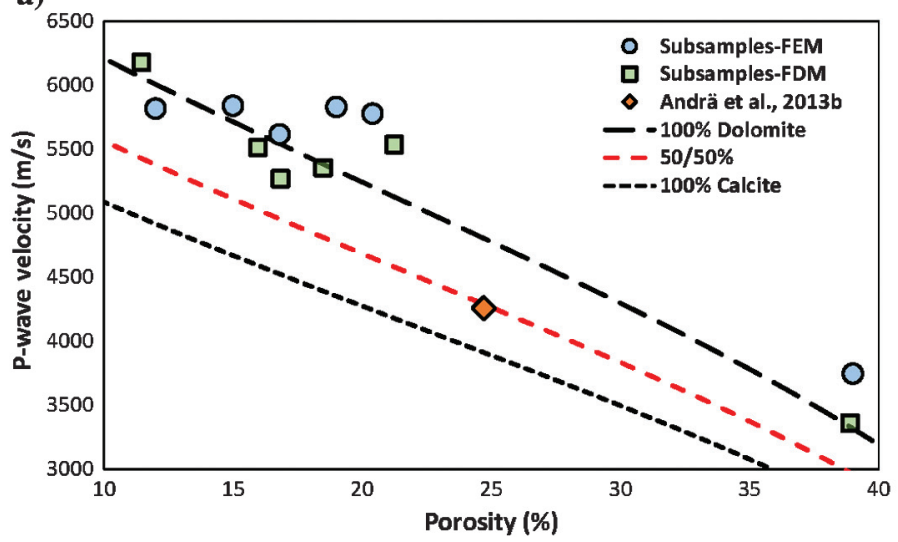

b)

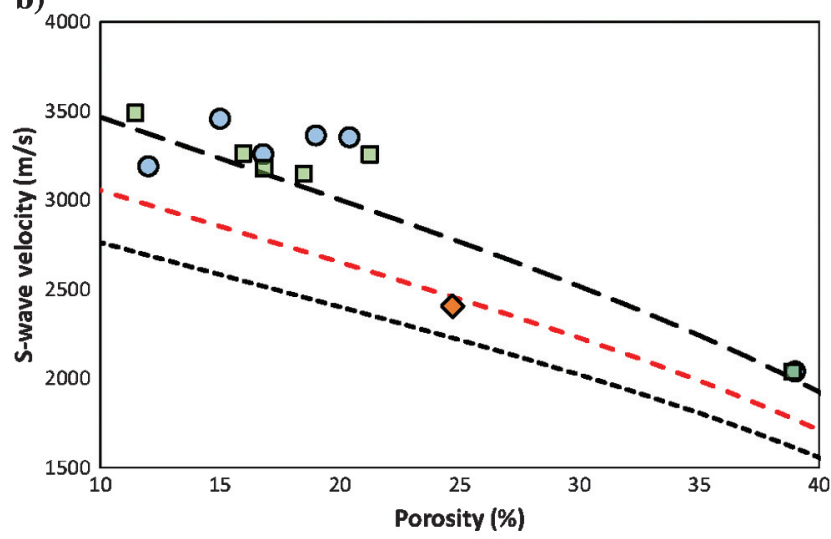

Figure 19. The same as Figure 14, but a 2D image of a thin section. 

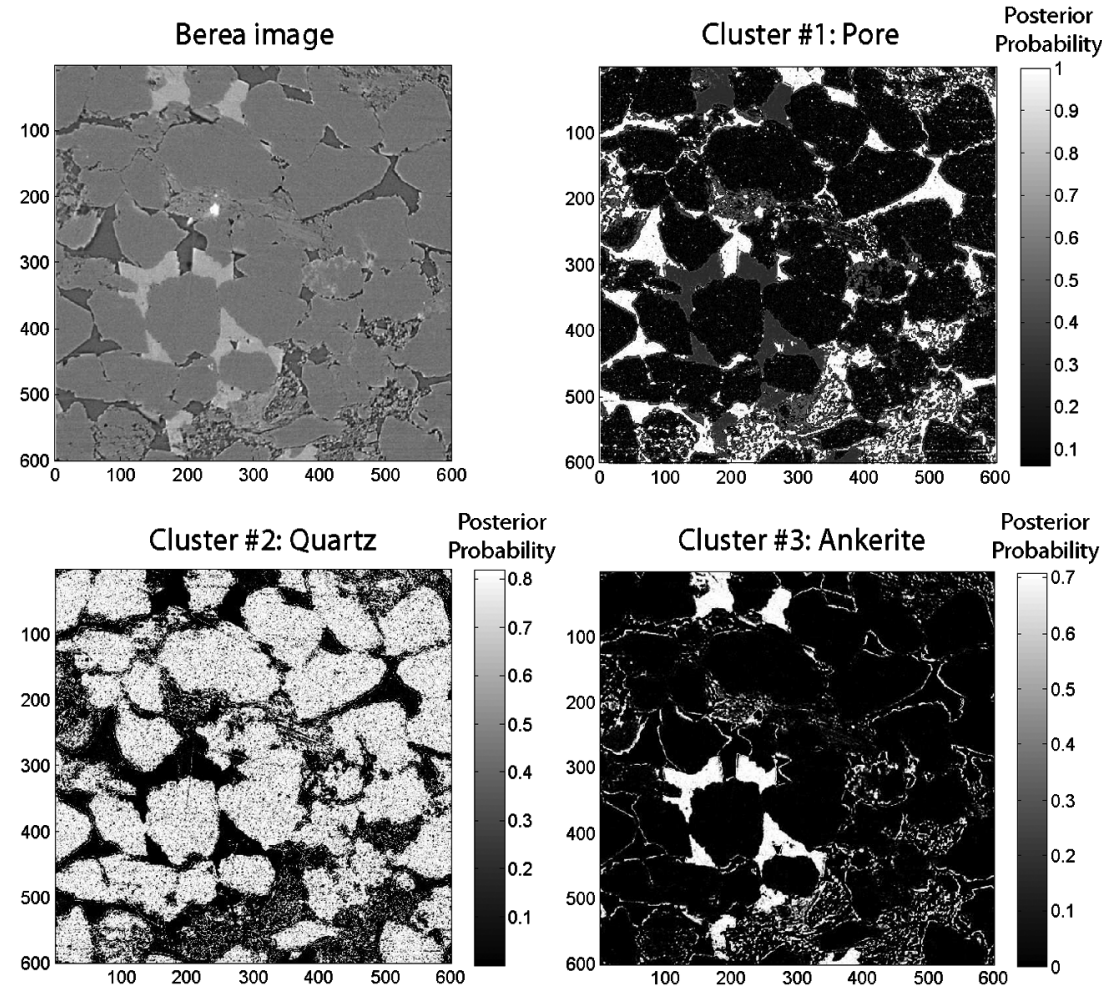

Figure 21. Berea sandstone image and posterior probabilities for $3 \mathrm{C}$ clustering using a Gaussian mixture distribution. Clusters are pore, first mineral (quartz), and second mineral (ankerite), respectively.

cal results. To evaluate this effect, a sample image with a size of $128 \times 128 \times 128$ pixels was selected from the Berea image cube. The porosity of this sample was calculated using both methods, and then, permeability and elastic velocities were accordingly computed and represented in Table 3 . These results show some differences, which is due to the existing differences of the calculated porosities. However, since main pore structures remain the same in two methods, numerical results do not show a significant variation. It should be noted that such small fluctuations due to seeking a global trend is not important. In other words, the final trend is not affected by these disparities. These two methods were used for several other subsamples, and they do not show an eloquent difference.

To evaluate the reconstruction models, realization models, pore network modeling, and autocorrelation functions were used. Results showed that reconstructed subsamples are valid enough to be as a representative of real rock medium. Moreover, simulation and computation of elastic-wave velocities using two static and dynamic methods revealed that, almost in all cases, subsamples can be considered as RVE. They also obey rock physical trends consistent with reference trends of the rock, which means this method can efficiently simulate valid rock physical parameters. Permeability results of Berea sample showed two different trends. In fact, bimodality of pore shape and throat size (fracture patterns and vuggy-type porosity) caused non-RVE subsamples for computing permeability resulted in bitrending. Finally, resampling the image, in this case, produced RVE subimages with more representative patterns. Then, more reliable permeability and trends were produced.

Selecting representative 2D images and subimages is also a significant issue. Since a $2 \mathrm{D}$ thin section is a very small part of the rock sample, it must be representative enough. From this point of view, using several thin sections or 2D images can be helpful to capture more real scenarios of the real rock structures. This problem is somehow the same as standard DRP because, there also, a millimeter sample must be chosen. Subimage selection can also be problematic because all subimages produced by one 2D image are definitely not proper and representative of real structure. Therefore, users should select those subimages with proper pore pattern.

Beside all of the various examples in this paper, most of the current DRP methods do not account for nonstationarity, which is very common in the reservoirs (Tahmasebi and Sahimi, 2015a, 2015b). Unlike the stationary porous media, statistical properties in the nonstationary media vary spatially, and thus, no representative sample can be defined to describe the variability of whole sample. As a result, the predicted rock physical properties are biased because these geological facts are ignored. This issue has been studied and its results will be reported in future papers.

\section{CONCLUSIONS}

An efficient alternative method for DRP procedure was proposed in this paper. This method, which is a modification of standard DRP, uses a $2 \mathrm{D}$ image instead of 3D $\mu \mathrm{CT}$-scan image. First, it divides a large high-resolution 2D image into subimages. Next, different stochastic 3D samples using the CCSIM algorithm are generated. These subsamples are segmented and then rock physical parameters are calculated. Subsampling, in this study, allows us to find rock physical trends. This idea was implemented on two DRP benchmark data: Berea sandstone and Grosmont carbonate. To show the ability of this method, a 2D thin section image of Grosmont formation was also used as the input image. Eventually, various rock physical parameters were computed.

According to the obtained results, elastic-wave velocities were consistent with reference rock trends in all cases. These results, in some cases, were slightly higher than the actual values in both computational methods. This can be related to image resolution while the larger voxel size, the stiffer sample. If the resolution of the image is low, microporosities, microcracks, and grain-to-grain contacts, decreasing elastic moduli of the rock sample, are not captured and they will be considered as grain. This is obviously seen in thin section results, which, although they obey the reference trends, their values are similar to the stiff sand model with $100 \%$ dolomite mineral composition.

Finally, it can be concluded that the proposed method is valid and effective enough to simulate the same results as standard DRP. Therefore, it can be used where a $\mu$ CT-scanner is not available and/or as an efficient alternative method instead of expensive and time-consuming DRP.

\section{ACKNOWLEDGMENTS}

We would like to thank M. Blunt (Imperial College) and his group for permission to use their pore network modeling codes. We also thank E. Saenger (ETH, Zurich) for his time and patience 
for simulating our subsamples using the dynamic method. We also wish to thank the associate editor and five anonymous reviewers of this manuscript for their constructive and valuable comments that improved the initial manuscript.

\section{REFERENCES}

Andrä, H., N. Combaret, J. Dvorkin, E. Glatt, J. Han, M. Kabel, Y. Keehm, F. Krzikalla, M. Lee, C. Madonna, M. Marsh, T. Mukerji, E. H. Saenger, R. Sain, N. Saxena, S. Ricker, A. Wiegmann, and X. Zhan, 2013a, Digital rock physics benchmarks - Part I: Imaging and segmentation: Computers and Geosciences, 50, 25-32, doi: 10.1016/j.cageo.2012.09.005.

Andrä, H., N. Combaret, J. Dvorkin, E. Glatt, J. Han, M. Kabel, Y. Keehm, F Krzikalla, M. Lee, C. Madonna, M. Marsh, T. Mukerji, E. H. Saenger, R. Sain, N. Saxena, S. Ricker, A. Wiegmann, and X. Zhan, 2013b, Digital rock physics benchmarks - Part II: Computing effective properties: Computers and Geosciences, 50, 33-43, doi: 10.1016/j.cageo.2012.09.008.

Blunt, M. J., B. Bijeljic, H. Dong, O. Gharbi, S. Iglauer, P. Mostaghimi, A Paluszny, and C. Pentland, 2013, Pore-scale imaging and modelling: Advances in Water Resources, 51, 197-216, doi: 10.1016/j.advwatres 2012.03.003.

Box, G. E. P., G. M. Jenkins, and G. C. Reinsel, 1994, Time series analysis: Forecasting and control (3rd ed.): Prentice-Hall.

Ceron, M. R., J. F. Martinez, E. Diaz, J. Walls, and C. Sisk, 2013, Digital rock physics for reservoir characterization: Proceedings of the 13th International Congress of the Brazilian Geophysical Society, 1134-1136.

Derzhi, N., C. Sisk, and A. Kalam, 2011, Application of digital rock physics for geophysical rock properties: Proceedings of the 12th International Congress of the Brazilian Geophysical Society, 1472-1475.

Dong, H., and M. J. Blunt, 2009, Pore-network extraction from micro- computerized-tomography images: Physical Review E, 80, 036307, doi: 10 1103/PhysRevE.80.036307.

Dvorkin, J., and N. Derzhi, 2012, Rules of upscaling for rock physics transforms: Composites of randomly and independently drawn elements: Geophysics, 77, no, 3, WA129-WA139, doi: 10.1190/geo2011-0268.1.

Dvorkin, J., N. Derzhi, E. Diaz, and Q. Fang, 2011, Relevance of computational rock physics: Geophysics, 76, no. 5, E141-E153, doi: 10.1190/ geo2010-0352.1.

Dvorkin, J., and A. Nur, 1996, Elasticity of high-porosity sandstones: Theory for two North Sea data sets: Geophysics, 61, 1363-1370, doi: 10.1190/1 .1444059 .

Ferreol, B., and D. H. Rothman, 1995, Lattice-Boltzmann simulations of flow through Fontainebleau sandstone: Transport in Porous Media, 20, 3-20, doi: 10.1007/BF00616923.

Gao, M. L., X. H. He, Q. Z. Teng, C. Zuo, and D. D. Chen, 2015, Reconstruction of three-dimensional porous media from a single two-dimensional image using three-step sampling: Physical Review E, 91, 013308, doi: 10 .1103/PhysRevE.91.013308

Garboczi, E. J., and A. Day, 1995, Algorithm for computing the effective linear elastic properties of heterogeneous materials: Three dimensional results for composites with equal phase Poisson ratios: Journal of the Mechanics and Physics of Solids, 43, 1349-1362, doi: 10.1016/0022-5096 (95)00050-S.

Jouini, M. S., S. Vega, and A. Al-Ratrout, 2014, Numerical estimation of carbonate rock properties using multiscale images: Geophysical Prospecting, 63, 405-421, doi: 10.1111/1365-2478.12156.

Karimpouli, S., H. Hassani, M. Nabi-Bidhendi, H. Khoshdel, and A. Malehmir, 2013, Application of probabilistic facies prediction and estimation of rock physics parameters in a carbonate reservoir from Iran: Journal of Geophysics and Engineering, 10, 015008, doi: 10.1088/1742-2132/10/1/ 015008.

Keehm, Y., T. Mukerji, and A. Nur, 2003, Permeability prediction from thin sections: 3D reconstruction and lattice-Boltzmann flow simulation: Geophysical Research Letters, 31, L04606.

Latief, F. D. E., B. Biswal, U. Fauzi, and R. Hilfer, 2010, Continuum reconstruction of the pore scale microstructure for Fontainebleau sandstone, Physica A, 389, 1607-1618, doi: 10.1016/j.physa.2009.12.006.

Liang, Z., M. A. Ioannidis, and I. Chatzis, 2000, Permeability and electrical conductivity of porous media from 3D stochastic replicas of the microstructure: Chemical Engineering Science, 55, 5247-5262, doi: 10.1016/ S0009-2509(00)00142-1.

Liubis, L. A., and Z. Z. T. Harith, 2014, Pore type classification on carbonate reservoir in offshore Sarawak using rock physics model and rock digital images: IOP conf. series: Earth and Environmental Science, 19, 012003.

Lopez, O., A. Mock, P. E. Øren, H. Long, A. Kalam, V. Vahrenkamp1, M. Gibrata, S. Seraj, S. Chacko, M. Al-Hammadi, H. Al-Hosni, H. Sahn, and A. Vizamora, 2012, Validation of fundamental carbonate reservoir core properties using digital rock physics: International Symposium of the Society of Core Analysts, SCA2012-19.

Madonna, C., B. S. G. Almqvist, and E. H. Saenger, 2012, Digital rock physics: Numerical prediction of pressure-dependent ultrasonic velocities using $\mu \mathrm{CT}$ imaging: Geophysical Journal International, 189, 1475-1482, doi: $10.1111 /$ gij.2012.189.issue-3.

McLachlan, G., and D. A. Peel, 2000, Finite mixture models: Wiley.

Mostaghimi, P., M. J. Blunt, and B. Bijeljic, 2013, Computations of absolute permeability on micro-CT images: Mathematical Geosciences, 45, 103125, doi: 10.1007/s11004-012-9431-4.

Narváez, A., T. Zauner, F. Raischel, R. Hilfer, and J. Harting, 2010, Quantitative analysis of numerical estimates for the permeability of porous media from lattice-Boltzmann simulations: Journal of Statistical Mechanics, 11, P11026.

Okabe, H., and M. J. Blunt, 2004, Prediction of permeability for porous media reconstructed using multiple-point statistics: Physical Review E, 70, 066135, doi: 10.1103/PhysRevE.70.066135.

Okabe, H., and M. J. Blunt, 2005, Pore space reconstruction using multiplepoint statistics: Journal of Petroleum Science and Engineering, 46, 121137, doi: 10.1016/j.petrol.2004.08.002.

Okabe, H., and M. J. Blunt, 2007, Pore space reconstruction of vuggy carbonates using microtomography and multiple-point statistics: Water Resource Reserve, 43, W12S02.

Ringstad, C., E. Westphal, A. Mock, M. Al-Hammadi, and Z. Kalam, 2013, Elastic properties of carbonate reservoir rocks using digital rock physics: 75th International Conference and Exhibition incorporating, EAGE, SPE EUROPEC 2013.

Russel-Houston, J., and J. Y. Yuan, 2010, The potential of Grosmont carbonates and the path for realizing it: In SPE Technical Luncheon.

Saenger, E. H., 2008, Numerical methods to determine effective elastic properties: International Journal of Engineering Science, 46, 598-605, doi: 10 $.1016 /$ j.ijengsci.2008.01.005.

Saenger, E. H., F. Enzmann, Y. Kehhm, and H. Steeb, 2011, Digital rock physics: Effect of fluid viscosity on effective elastic properties: Journal of Applied Geophysics, 74, 236-241, doi: 10.1016/j.jappgeo.2011.06.001.

Saenger, E. H., N. Gold, and S. A. Shapiro, 2000, Modeling the propagation of elastic waves using a modified finite-difference grid: Wave Motion, 31, 77-92, doi: 10.1016/S0165-2125(99)00023-2.

Saenger, E. H., O. S. Krüger, and S. A. Shapiro, 2006, Effective elastic properties of fractured rocks: Dynamic versus static considerations: International Journal of Fractures, 139, 569-576, doi: 10.1007/s10704-006-0105-4.

Saenger, E. H., C. Madonna, N. Tiaso, and B. Quintal, 2014, Towards a representative rock model from a $\mu \mathrm{CT}$ image: Second EAGE Workshop on Rock Physics - Rock Physics: Integration \& Beyond.

Sezgin, M., and B. Sankur, 2004, Survey over image thresholding techniques and quantitative performance evaluation: Journal of Electronic Imaging, 13, 146-165, doi: 10.1117/1.1631315.

Sochi, T., and M. J. Blunt, 2008, Pore-scale network modeling of Ellis and Herschel-Bulkley fluids: Journal of Petroleum Science and Engineering, 60, 105-124, doi: 10.1016/j.petrol.2007.05.009.

Tahmasebi, P., A. Hezarkhani, and M. Sahimi, 2012, Multiple-point geostatistical modeling based on the cross-correlation functions: Computational Geosciences, 16, 779-797, doi: 10.1007/s10596-012-9287-1.

Tahmasebi, P., F. Javadpour, and M. Sahimi, 2015a, Three-dimensional stochastic characterization of shale SEM images: Transport in Porous Media, 110, 521-531, doi: 10.1007/s11242-015-0570-1.

Tahmasebi, P., F. Javadpour, and M. Sahimi, 2015b, Multiscale and multiresolution modeling of shales and their flow and morphological properties: Scientific Reports, 5, 16373, doi: 10.1038/srep16373.

Tahmasebi, P., and M. Sahimi, 2012, Reconstruction of three-dimensional porous media using a single thin section: Physical Review E, 85, 066709, doi: 10.1103/PhysRevE.85.066709.

Tahmasebi, P., and M. Sahimi, 2013, Cross-correlation function for accurate reconstruction of heterogeneous media: Physical Review Letters, 110, 078002, doi: 10.1103/PhysRevLett.110.078002.

Tahmasebi, P., M. Sahimi, and J. Caers, 2014, MS-CCSIM: Accelerating pattern-based geostatistical simulation of categorical variables using a multi-scale search in Fourier space: Computer and Geosciences, 67, 75-88, doi: 10.1016/j.cageo.2014.03.009.

Tahmasebi, P., and M. Sahimi, 2015a, Reconstruction of nonstationary disordered materials and media: Watershed transform and cross-correlation function: Physical Review E, 91, 032401, doi: 10.1103/PhysRevE.91.032401.

Tahmasebi, P., and M. Sahimi, 2015b, Geostatistical simulation and reconstruction of porous media by a cross-correlation function and integration of hard and soft data: Transport in Porous Media, 107, 871-905, doi: 10 $.1007 / \mathrm{s} 11242-015-0471-3$

Talukdar, M. S., and O. Torsaeter, 2002, Reconstruction of chalk pore networks from 2D backscatter electron micrographs using a simulated annealing technique: Journal of Petroleum Science and Engineering, 33, 265-282, doi: 10.1016/S0920-4105(02)00148-1. 